\title{
Kompetenzverstoß der Europäischen Union: die Sanktionierung der Eurostaaten im Rahmen der Excessive Imbalance Procedure
}

von Florian Rödl

Der Beitrag berichtet über ein Gutachten zur Kompetenz der Europäischen Union zum Erlass der Verordnung (EU) Nr. 1174/2011 über Durchsetzungsmaßnahmen zur Korrektur übermäßiger Ungleichgewichte im Eurowährungsgebiet. Als Ergebnis ist festzuhalten, dass die Verordnung die Grenzen der Unionskompetenzen überschreitet. Die Union ist weder berechtigt, im umfassenden Bereich der Wirtschaftspolitik für die Mitgliedstaaten verbindliche Regelungen zu treffen, noch darf generell die materielle Entscheidungsverantwortung sekundärrechtlich durch die Beschlussregel der ,umgekehrten Mehrheit“ vom Rat auf die Kommission verschoben werden.

This report summarises the results of a legal opinion on the question whether the adoption of Regulation (EU) No. 1174/2011 is covered by the competences of the European Union. The findings indicate a negative answer: first, it is beyond the competences of the Union to adopt binding regulations in the broad field of Member States' economic policies. Second, substantive decision-making responsibility may not be shifted from the Council to the Commission via secondary legislation by introducing a "reverse majority" voting procedure.

\section{Hintergrund, Kontext und Gehalt der maßgeblichen Verordnung (EU) Nr. 1174/2011}

Im Zuge der Euro-Krise erfolgte eine Stärkung der so genannten economic governance in der Union. Die Stärkung bestand nicht zuletzt in der Einführung der makroökonomischen Überwachung der volkswirtschaftlichen Entwicklung in den Mitgliedstaaten seitens der Union, einschließlich eines Verfahrens bei übermäßigem makroökonomischem Ungleichgewicht (sogenannte excessive imbalance procedure, EIP). Von diesem Verfahren verspricht sich die europäische Politik eine wirksame Bekämpfung der makroökonomischen Ungleichgewichte in der Eurozone, wie sie sich etwa in den hohen Leistungsbilanzüberschüssen

Anmerkung der Schriftleitung: Das hier summierte Gutachten wurde im Auftrag des Hugo-SinzheimerInstituts von Jürgen Bast und Florian Rödl erstattet. 
und -defiziten einer Reihe von Mitgliedstaaten finden. Der EIP kommt daher in der Strategie der Union zur nachhaltigen Bewältigung der Euro-Krise eine zentrale Rolle zu: Nur wenn sie hinreichende Steuerungseffekte entfaltet, gewinnt der generelle Ansatz der Wirtschafts- und Währungsunion neue Glaubwürdigkeit, trotz Fehlens einer Politischen Union die wirtschaftliche Konvergenz der Mitgliedstaaten als zentrale Funktionsbedingung des Euro sichern zu wollen.

Den rechtlichen Rahmen des Verfahrens der EIP, das im Groben dem Verfahren bei übermäßigem Haushaltsdefizit nach Art. 126 AEUV nachgebildet ist, bilden zwei förmlich voneinander unabhängige Verordnungen des Europäischen Parlaments und des Rates. Die erste ist die Verordnung (EU) Nr. 1176/2011 „über die Vermeidung und Korrektur makroökonomischer Ungleichgewichte", 1 die auf sämtliche Mitgliedstaaten Anwendung findet (nachfolgend: KorrekturVO), die zweite ist die Verordnung (EU) Nr. 1174/2011 „über Durchsetzungsmaßnahmen zur Korrektur übermäßiger Ungleichgewichte im Eurowährungsgebiet" ${ }^{\text {" }}$, die nur die Mitglieder der Eurozone betrifft (nachfolgend: DurchsetzungsVO). Die KorrekturVO ist ein Instrument zur intensiveren Koordinierung der Wirtschaftspolitik der Mitgliedstaaten der EU. Ein Mitgliedstaat, dessen Volkwirtschaft ein übermäßiges Ungleichgewicht aufweist, muss Kommission und Rat nach deren Maßgaben einen Maßnahmenplan zur Korrektur dieser ungleichgewichtigen Situation vorlegen (Art. 7 f. KorrekturVO). Legt der Mitgliedstaat einen aus Sicht von Kommission und Rat hinreichenden Korrekturmaßnahmenplan vor, wird dem Mitgliedstaat die Durchführung dieses Planes empfohlen. Den Mitgliedstaat trifft aber keine rechtliche Pflicht, diese Empfehlung einzuhalten. Eine etwaige Nichteinhaltung wird lediglich durch Ratsbeschluss festgehalten (Art. 10 Abs. 4 KorrekturVO). Die DurchsetzungsVO, die nur auf die Mitglieder der Eurozone anwendbar ist, schließt an dieser Stelle an. Deren regulative Pointe liegt darin, es zu ermöglichen, jeden Euro-Mitgliedstaat mit Sanktionen in Gestalt von unverzinslichen Einlagen und Geldbußen zu belegen, der seinen Korrekturmaßnahmenplan nicht einhält oder der zum wiederholten Male einen aus Sicht von Kommission und Rat unzureichenden Maßnahmenplan vorlegt (Art. 3 Abs. 1 und 2 DurchsetzungsVO).

Die wesentliche Besonderheit der EIP liegt darin, dass sich die Überwachung auf die mitgliedstaatliche Wirtschaftspolitik in ihrer Breite erstreckt und damit auch 
Sachbereiche erfasst, die einem regelnden Zugriff der Union auf Basis ihrer regulativen Kompetenzen weitgehend verschlossen sind. In Folge der EIP können also die verbliebenen Gestaltungsspielräume der Mitgliedstaaten erheblich einschränkt werden, wenn es aus Sicht der zuständigen Organe der EU zu übermäßigen makroökonomischen Ungleichgewichten gekommen ist. Der mitgliedstaatliche Korrekturmaßnahmenplan beschränkt sich nicht auf Politikbereiche und Regelungsgegenstände, in denen die Union eigene regulative Kompetenzen innehat, etwa den Bereich des Wettbewerbs oder die Regelungsgegenstände mit Auswirkungen auf das Funktionieren des Binnenmarktes. Wie in der Begründungserwägung 20 der KorrekturVO ausdrücklich festgehalten, ist der Mitgliedstaat vielmehr gehalten, sämtliche administrativen, legislativen und sozialpartnerschaftlichen Handlungsmöglichkeiten in den von ihm vorzulegenden Maßnahmenplan einzubeziehen. Indem die Einhaltung des Plans aufgrund der DurchsetzungsVO sanktionsbewehrt wird, erhält die Unionsebene in diesem spezifischen Modus der Sanktion ein verbindliches Instrument der Einflussnahme auf alle Bereiche mitgliedstaatlicher Politik, die im Rahmen der Bewältigung makroökonomischer Ungleichgewichte eine Rolle spielen können.

\section{Ergebnisse rechtlicher Begutachtung}

Das hier summierte Gutachten konzentriert sich auf die Frage, ob die Verabschiedung der DurchsetzungsVO von den Kompetenzen der Union gedeckt ist. Nicht geprüft wurde die Frage, ob der festgestellte Kompetenzverstoß dasjenige qualifizierte $\mathrm{Ma} ß$ erreicht, um auch vor dem Bundesverfassungsgericht erfolgreich gerügt zu werden. Die wesentlichen Ergebnisse der Begutachtung werden im Folgenden referiert.

\section{Keine Kompetenz zur Regelung von Sanktionsbefugnissen des Rates im Bereich der Wirtschaftspolitik}

Eingangs der Prüfung war zu gewärtigen, dass die Kompetenzgrundlage in der DurchsetzungsVO offenbar nicht korrekt angegeben ist. Europäisches Parlament und Rat stützen sich auf „Artikel 136 in Verbindung mit Artikel 121 Absatz 6“. Art. 136 AEUV enthält jedoch lediglich eine Kompetenz des Rates. Insofern sollte die DurchsetzungsVO mutmaßlich auf Art. 121 Abs. 6 i.V.m. Art. 136 AEUV gestützt werden. Denn nur dann ist immerhin die Organkompetenz von Europäischem Parlament und Rat zum Erlass der DurchsetzungsVO nicht von vornherein zu verneinen. 
Art. 121 Abs. 6 AEUV ermächtigt Europäisches Parlament und Rat, im ordentlichen Gesetzgebungsverfahren die Einzelheiten des Verfahrens multilateraler Überwachung der mitgliedstaatlichen Wirtschaftspolitiken nach Art. 121 Abs. 3 und 4 AEUV festzulegen. Die multilaterale Überwachung besteht aus einer Abfolge aus Kommissionsberichten sowie Bewertungen, unverbindlichen Empfehlungen und Entscheidungen über eine Veröffentlichung dieser Empfehlungen. Sanktionen können in diesem Rahmen nicht erlassen werden. Es ist darum offenkundig, dass Art. 121 Abs. 6 AEUV allein die Regelung von Sanktionen, wie sie die DurchsetzungsVO vorsieht, nicht tragen würde. Daher kommt es entscheidend auf die Frage an, ob Art. 136 AEUV eine Ermächtigung des Rates einschließt, gegenüber den Mitgliedstaaten Sanktionen zu verhängen. Deren Einzelheiten könnten dann jedenfalls vertretbarer Weise von Europäischem Parlament und Rat auf Basis von Art. 121 Abs. 6 AEUV festgelegt werden.

Der maßgebliche Teil des Art. 136 AEUV - Abs. 1, v.a. lit. b), - lautet wörtlich:

Im Hinblick auf das reibungslose Funktionieren der Wirtschafts- und Währungsunion erlässt der Rat für die Mitgliedstaaten, deren Währung der Euro ist, Maßnahmen nach den einschlägigen Bestimmungen der Verträge und dem entsprechenden Verfahren unter den in den Artikeln 121 und 126 genannten Verfahren, mit Ausnahme des in Artikel 126 Absatz 14 genannten Verfahrens, um $(\ldots)$

b) für diese Staaten Grundzüge der Wirtschaftspolitik auszuarbeiten, wobei darauf zu achten ist, dass diese mit den für die Union angenommenen Grundzügen der Wirtschaftspolitik vereinbar sind, und ihre Einhaltung zu überwachen.

Diese Regelung dürfte dem unbefangenen Leser zunächst reichlich unverständlich erscheinen. Die sorgfältige Auslegung fördert jedoch einen klaren Sinn zutage.

Die maßgebliche Kompetenz des Rates, gegenüber Mitgliedstaaten auch Sanktionen zu verhängen, könnte sich allein aus dem letzten Halbsatz der Vorschrift ergeben, der zufolge der Rat mit Wirkung für die Mitgliedstaaten auch Maßnahmen ergreifen kann, um die Einhaltung der Grundzüge der Wirtschaftspolitik ,zu überwachen“. Denn eine Ermächtigung zu Überwachungsmaßnahmen könnte an sich, jedenfalls auf der Grundlage gewöhnlichen Sprachgebrauchs, Sanktionen einschließen.

Ein Vergleich der verschiedenen und gleichermaßen verbindlichen Sprachfassungen der Vorschrift fördert jedoch zunächst zutage, dass dieser Ansatzpunkt im Wortlaut sich zwar in der deutschen Fassung, nicht aber in der englischen und der französischen Fassung der Vorschrift findet. In englischer und französischer 
Fassung beschränkt sich die zusätzliche Ermächtigung des Rates auf die Verabschiedung besonderer Grundzüge der Wirtschaftspolitik. Die Überwachung ihrer Einhaltung ist nicht durch weitere zusätzliche Maßnahmen sicher zu stellen, sondern sie soll offenbar im Rahmen multilateraler Überwachung nach Art. 121 Abs. 3 und 4 AEUV erfolgen, Art. 136 Abs. 1 b) enthält insofern nur einen deklarativen Verweis.

Doch auch der Wortlaut der deutschen Fassung führt trotz des genannten Ansatzpunktes am Ende zum gleichen Ergebnis. Akte der Unionsorgane - im hiesigen Zusammenhang: eine Sanktionsmaßnahme des Rates - müssen stets in den im Vertrag selbst festgelegten Verfahren angenommen werden. Allgemeine Vorschriften hierzu finden sich in den Art. 293-296 AEUV, im Übrigen sind die Annahmeverfahren im Zusammenhang mit den einzelnen Ermächtigungen geregelt. So ist es auch bei Art. 136 Abs. 1 AEUV: Die Vorschrift verweist für die Regelung der Verfahren, nach denen der Rat seine Maßnahmen unter Art. 136 Abs. 1 b) AEUV anzunehmen hat, auf Art. 121 AEUV (der ebenfalls enthaltene Verweis auf Art. 126 AEUV bezieht sich ausschließlich auf Ratsmaßnahmen nach Art. 136 Abs. 1 a) AEUV zur verstärkten Koordinierung und Überwachung der Haushaltsdisziplin in der Eurozone). Wie oben bereits festgehalten, findet sich in Art. 121 Abs. 3 und 4 AEUV aber keine Ermächtigung des Rates zu Sanktionen, sondern eben nur zu Bewertungen, Empfehlungen und Entscheidungen über deren Veröffentlichung. Entsprechend regelt Art. 121 AEUV auch nur für diese Maßnahmen die Verfahren zu ihrer Annahme. Enthielte Art. 136 Abs. 1 b) tatsächlich eine Ermächtigung zu Sanktionen, ginge für das Annahmeverfahren der Verweis auf Art. 121 AEUV ins Leere. Das kann nicht richtig sein. Darum ist nach dem Wortlaut von Art. 136 Abs. 1 b) AEUV selbst auf der Basis seiner deutschen Fassung der Erlass von Sanktionen des Rates gegenüber einzelnen Mitgliedstaaten ausgeschlossen.

Dieses Ergebnis wird durch die Entstehungsgeschichte der Vorschrift gestützt. Art. 136 AEUV geht auf die Arbeit des Europäischen Konvents am Entwurf eines Vertrags für die Verfassung Europas zurück. Der für die deutsche Wortlautauslegung ausschlaggebende Verweis auf die Annahmeverfahren in Art. 121 AEUV wurde indessen erst im Zuge der Arbeiten einer Arbeitsgruppe von Rechtsexperten der Regierungskonferenz eingefügt. Im gleichen Zusammenhang wurde auch erst ausdrücklich festgehalten, dass die Handlungsermächtigung in Art. 136 Abs. 1 AEUV dem Rat erteilt werden sollte. Der von der Expertengruppe gegebenen Begründung lässt sich entnehmen, dass der für die Annahmeverfahren gegebene Verweis auf Art. 121 AEUV jene für die unter Art. 136 Abs. 1 
AEUV zulässigen Maßnahmen vollständig erfassen sollte. Vor diesem Hintergrund ist aus Sicht der Entstehungsgeschichte kein Raum für zusätzliche Maßnahmen wie etwa Sanktionen, für die nicht nur seitens des Konvents keine Annahmeverfahren vorgesehen gewesen wären, sondern welche auch die Expertengruppe im Rahmen ihrer eingehenden juristisch-redaktionellen Überarbeitung des Konventsentwurfs nicht ergänzt hätte, obwohl sich die Gruppe mit dem Problem der im Konventsentwurf noch ungeregelten Annahmeverfahren für die Ratsmaßnahmen unter Art. 136 Abs. 1 AEUV gezielt befasst hatte.

Systematische Gesichtspunkte sprechen ebenfalls für das Ergebnis der Wortlautauslegung. Art. 136 AEUV, obgleich in einem gesonderten Kapitel mit Sonderbestimmungen für die Eurozone, gehört systematisch zu den Vorschriften zur Wirtschaftspolitik (Art. 120-127 AEUV). Im Bereich der Wirtschaftspolitik kommt der Union eine in der zu Beginn des AEU-Vertrages festgehaltene Typologie der Kompetenzen gesondert geführte Koordinierungskompetenz zu (Art. 2 Abs. 3, Art. 5 AEUV). Für diesen Kompetenztypus ist kennzeichnend, dass die Union zwar naturgemäß die Koordinierung der jeweiligen mitgliedstaatlichen Politik auf europäischer Ebene verbindlich regelt, nicht aber die mitgliedstaatliche Politik selbst. Hierin besteht der wesentliche Unterschied zu den regulativen Kompetenztypen der ausschließlichen und geteilten Zuständigkeit (Art. 3 und 4 AEUV). Ausnahmen sind zwar durch diese Typisierung der Kompetenzen in Art. 2 ff. AEUV nicht ausgeschlossen. Den Ausschlag gibt am Ende immer die maßgebliche Kompetenznorm (vgl. Art. 2 Abs. 6 AEUV). Doch ohne ausdrückliche Regelungen - wie sie im Bereich der Wirtschaftspolitik etwa in Gestalt von Art. 126 AEUV vorliegen - spricht die Systematik dafür, dass sich eine fragliche Kompetenznorm im Rahmen des ihr entsprechenden allgemeinen Kompetenztypus hält. Das bedeutet für die Auslegung von Art. 136 Abs. 1 b) AEUV, dass die Vorschrift mangels einer anderslautenden eindeutigen Regelung den Rat nicht zu regulativen Maßnahmen und also auch nicht zu Sanktionen gegenüber den Mitgliedstaaten ermächtigt.

Schließlich weist auch die teleologische Auslegung in dieselbe Richtung. Wenn Art. 136 AEUV so gelesen würde, dass die dort genannten „Maßnahmen“ des Rates auch Sanktionen einschlössen, dann müsste die Vorschrift darüber hinaus auch Verordnungen und Richtlinien tragen. Da die sachliche Reichweite des Bereichs der Wirtschaftspolitik denkbar weit ist und insbesondere auch die Fiskalpolitik, die Steuerpolitik sowie die Arbeits- und Sozialpolitik einschließt, würde dies bedeuten, dass Art. 136 Abs. 1 b) AEUV der Union (und innerhalb der Union dem Rat!) mit Wirkung für die Eurozone eine umfassende und nach 
Form und Richtung nicht weiter qualifizierte Gesetzgebungskompetenz für all jene Politikbereiche zugesprochen hätte. Mit Art. 136 Abs. 1 b) AEUV wäre also das differenzierte Kompetenzgefüge in eben diesen Bereichen für die Mitglieder der Eurozone mit einem Schlag überschrieben worden. Diese Konsequenz einer solchen Auslegung von Art. 136 Abs. 1 b) AEUV liegt an der Grenze des Absurden. Folglich wird man Art. 136 Abs. 1 b) AEUV den entsprechenden Zweck einer weit reichenden Unterminierung der Kompetenzordnung mit Wirkung für die Eurozone nicht unterlegen können. Dies gilt vor allem vor dem Hintergrund, dass dieser dann exorbitante Integrationssprung bis heute unbekannt geblieben und offenbar selbst den politischen Akteuren der Vertragsrevision von Lissabon sowie einer Vielzahl sachkundiger Beobachter entgangen wäre.

Das Gutachten kommt nach alledem zu dem Ergebnis: Art. 136 Abs. 1 b) AEUV enthält keine Ermächtigung des Rates zur Verhängung von Sanktionen. Entsprechend durfte eine Verordnung von Europäischem Parlament und Rat auf der Basis von Art. 121 Abs. 6 i.V.m. 136 AEUV auch keine Sanktionierung der Mitgliedstaaten durch den Rat regeln.

\section{Keine Kompetenz zur Regelung der Beschlussfassung des Rates im Wege „umgekehrte Mehrheit“}

Auch wenn aufgrund des Kompetenzverstoßes die Rechtswidrigkeit der DurchsetzungsVO im Ganzen festgestellt ist, wird im hier referierten Gutachten gesondert die Regelung zur Beschlussfassung des Rates über die Sanktionen an die Mitgliedstaaten gem. Art. 3 Abs. 3 DurchsetzungsVO überprüft. Diese Regelung lautet wörtlich:

Werden die in den Absätzen 1 und 2 genannten Beschlüsse nicht binnen zehn Tagen nach ihrer Annahme durch die Kommission vom Rat mit qualifizierter Mehrheit abgelehnt, so gelten sie als vom Rat angenommen. Der Rat kann die Empfehlung mit qualifizierter Mehrheit ändern.

Auf diese Regelung wird in der (rechts-)politischen Diskussion bisweilen mit dem Ausdruck ,umgekehrte Mehrheit“ Bezug genommen. Sie zeichnet einerseits aus, dass sie den Sanktionsbeschluss formal eindeutig dem Rat zuweist. Bei einer gegen einen Sanktionsbeschluss erhobenen Klage wäre der Rat darum der richtige Beklagte. Aber andererseits muss sich der Rat als formeller Autor des Beschlusses nicht notwendiger Weise positiv für die Annahme des Beschlusses ausgesprochen haben. Es genügt, dass er der Empfehlung der Kommission nicht widersprochen hat. 
Vor diesem Hintergrund war zunächst festzustellen, dass Europäisches Parlament und Rat an dieser Stelle außerhalb ihrer Organzuständigkeit gehandelt haben. Die Festlegung der Beschlussfassungsregeln obliegt auf Basis des Grundsatzes der Selbstorganisationsbefugnis der Hauptorgane der Union jeweils dem betroffenen Organ allein. Dieser Grundsatz des Unionsrechts ist für den Rat in Gestalt von dessen Befugnis zum Erlass seiner Geschäftsordnung in Art. 240 Abs. 3 AEUV konkretisiert. Eine allgemeine Befugnis zur übergreifenden Regelung von Fragen des Organisationsrechts besitzen Europäisches Parlament und Rat auch in ihrer Rolle als ordentlicher Unionsgesetzgeber (vgl. Art. 294 AEUV) nicht. Es liegt demnach nicht in der Zuständigkeit von Europäischem Parlament und Rat, sondern es läge allenfalls in der Kompetenz des Rates allein, eine dem Vorbild von Art. 3 Abs. 3 DurchsetzungsVO entsprechende Beschlussfassungsregel zu verabschieden.

Zum Zweiten verstößt die Beschlussfassungsregel in Art. 3 Abs. 3 DurchsetzungsVO materiell gegen die primärrechtliche Regelung über die Beschlussfassung des Rates in Art. 16 Abs. 3 EUV. Dort ist festgelegt, dass der Rat seine Beschlüsse mit qualifizierter Mehrheit trifft, soweit ,in den Verträgen“ keine anderweitige Regelung getroffen ist. Mangels einer abweichenden Beschlussfassungsregel in Art. 136 Abs. 1 AEUV (Absatz 2 regelt allein die Stimmberechtigung der Ratsmitglieder) ist daher in diesem Zusammenhang die Beschlussfassung nach Art. 16 Abs. 3 EUV unmittelbar maßgeblich. Zwar spielt im Rahmen der Beschlussfassung nach Art. 3 Abs. 3 DurchsetzungsVO die qualifizierte Mehrheit durchaus eine Rolle. Doch der Rat muss nicht positiv einen Sanktionsbeschluss mit qualifizierter Mehrheit tragen, sondern er muss es negativ unterlassen, einem von der Kommission empfohlenen Sanktionsbeschluss zu widersprechen. Art. 16 Abs. 3 EUV verlangt aber nicht nur, dass die qualifizierte Mehrheit bei der Beschlussfassung des Rates irgendeine Rolle spielt, sondern die Vorschrift verlangt, dass die qualifizierte Mehrheit den Beschluss des Rates trägt. Insofern steht Art. 3 Abs. 3 DurchsetzungsVO in Widerspruch zu Art. 16 Abs. 3 EUV. Europäisches Parlament und Rat sind aber im Rahmen von Art. 121 Abs. 6 AEUV nicht befugt, die primärrechtlichen Regelungen zur Beschlussfassung im Rat im Bereich der multilateralen Überwachung nach Art. 121 Abs. 3 und 4 AEUV sekundärrechtlich abweichend zu regeln.

Schließlich ist festzustellen, dass Art. 3 Abs. 3 DurchsetzungsVO auch deswegen gegen Primärrecht verstößt, weil er das institutionelle Gleichgewicht unter den Unionsorganen materiell verschiebt. Das in Art. 13 Abs. 2 AEUV verankerte Prinzip des institutionellen Gleichgewichts besagt, dass jedes Organ nach Maß- 
gabe der vertraglich zugewiesenen Befugnisse handelt und diese horizontale Kompetenzverteilung durch Organhandeln, etwa den Erlass von Sekundärrecht, nicht verschoben werden darf. Zwar verbleibt mit Art. 3 Abs. 3 DurchsetzungsVO die formelle Entscheidungsverantwortung beim Rat. Materiell aber ist der Rat nur zur Ausübung eines befristeten Vetorechts berufen. Die substantielle Entscheidungsverantwortung liegt damit nicht mehr beim Rat, sondern bei der Kommission. Damit wird das vom Vertrag in Art. 136 Abs. 1 AEUV i.V.m. Art. 121 Abs. 3 und 4 AEUV statuierte Verhältnis von Rat und Kommission derart unterminiert, dass ein Verstoß gegen das institutionelle Gleichgewicht zu bejahen ist. $\mathrm{Zu}$ einer solchen Verschiebung waren Europäisches Parlament und Rat auf der Basis von Art. 121 Abs. 6 AEUV nicht ermächtigt.

$\mathrm{Zu}$ bemerken ist an dieser Stelle noch, dass sich die geprüfte Vorschrift in Art. 3 Abs. 3 DurchsetzungsVO auch in anderen Regelungswerken zur Stärkung der „economic governance" der Union findet, namentlich in Art. 10 Abs. 4 UAbs. 2 S. 1 der KorrekturVO, in Art. 1 Ziffer 9 und 13 der Verordnung (EU) Nr. 1175/2011 zur Änderung der Verordnung (EG) 1466/1997 des Rates über den Ausbau der haushaltspolitischen Überwachung und der Überwachung und Koordinierung der Wirtschaftspolitiken, ${ }^{3}$ sowie in Art. 4 Abs. 2 und Art. 6 Abs. 2 der Verordnung (EU) Nr. 1173/2011 über die wirksame Durchsetzung der haushaltspolitischen Überwachung im Euro-Währungsgebiet. ${ }^{4}$ Da sich die soeben wieder gegebene Argumentation nicht auf den Inhalt der in den genannten Verordnungen jeweils geregelten Beschlüsse des Rates bezog, sondern auf das Verfahren ihrer Annahme, sind die Ergebnisse unmittelbar auf diese übertragbar: Die zitierten Regelungen sind wegen Verstoßes gegen das Primärrecht rechtswidrig.

\section{Ertrag für Wissenschaft, legislative Praxis und verfassungs- politische Diskussion}

Das Gutachten sorgt für Klarheit mit Bezug auf die Reichweite von Art. 136 AEUV, der nach den Worten Ulrich Hädes von einigen bereits als „Wunderwaffe ${ }^{\text {“5 }}$ gepriesen worden war. Während in der deutschen Literatur zwar vor allem skeptische Stimmen zu den wichtigsten Fällen seiner jüngsten Inanspruchnahme

5 Häde, U.: Art. 136 AEUV - eine neue Generalklausel für die Wirtschafts- und Währungsunion?, in: Juristenzeitung 7/2011, 333-340, hier 333. 
zu hören waren, ${ }^{6}$ scheinen einige Autoren auch bereit, die jüngere Gesetzgebungspraxis der Unionsorgane auch rechtlich als maßgebend zumindest für die Zukunft anzusehen. Vor dem Hintergrund des hier referierten Gutachtens dürfte eine solche Herangehensweise als kaum mehr vertretbar erscheinen: Der Gesetzgeber der Union hat mit der DurchsetzungsVO die geltenden Grenzen seiner Kompetenzen klar überschritten. Diese Überschreitung kann künftige Wiederholungen vergleichbarer Kompetenzverstöße nicht legalisieren.

Positiver ausgedrückt liefert das Gutachten einen wesentlichen Beitrag dazu, im Bereich der sog. economic governance die Grenzen zu konturieren, innerhalb derer gegenwärtig politische und institutionelle Reformen von Regierung und Steuerung im Rahmen der Eurozone und als Reaktion auf die fortlaufende Krise möglich sind. Sollen diese Grenzen überschritten werden, müssen die legitimatorischen Mühen einer Vertragsänderung aufgewandt werden. Alternativ können die mitgliedstaatlichen Regierungen wie im Falle des Fiskalpaktes auf den Modus intergouvernementaler Nebenverfassung ausweichen, der freilich seinerseits nicht frei von kritischen Anfechtungen unterschiedlicher Art ist. ${ }^{7}$

Aber nicht nur mit Blick auf die Bewältigung der Euro-Krise, auch für die mitunter weit darüber hinaus weisenden Diskussionen über Reformen oder gar Transformationen der Union leistet das Gutachten einen hilfreichen Beitrag. Es ist in diesen Diskussionen sicherlich interessant und wichtig zu gewärtigen, dass etwa eine keynesianisch angelegte „demokratische EU-Wirtschaftsregierung“ mit umfassenden Kompetenzen im Bereich der Arbeits-, Sozial- und Steuerpolitik eine Vorstellung, die schon vor allem sozialdemokratische und gewerkschaftliche Kreise beschäftigt ${ }^{8}$ - nicht etwa dank Art. 136 Abs. 1 b) AEUV schon im Rahmen der bestehenden Verträge möglich wäre, sondern eine Vertragsänderung voraussetzt.

Schließlich liefert das Gutachten auch eine juristische Referenz, sollte im Rahmen der künftigen Verfahren zu makroökonomischen Ungleichgewichten ein Mitgliedstaat Klage vor dem EuGH gegen eine vom Rat auf Basis der DurchsetzungsVO verhängte Sanktion erheben. Dies ist nämlich - auch darüber gibt das hier referierte Gutachten Aufschluss - im Falle eines künftigen Sanktionsbe-

6 Häde, U., a.a.O.; Weber, A., Die Reform der Wirtschafts- und Währungsunion in der Finanzkrise, in: Europäische Zeitschrift für Wirtschaftsrecht 22 (2011), 935-940, hier: 936.

7 Vgl. den Beitrag von Frank Schorkopf in diesem Heft.

8 S. etwa Collignon, S.: Demokratische Anforderungen an eine europäische Wirtschaftsregierung, 2010, hier 12ff.; Busch, K.: Scheitert der Euro?, 2010, hier 43. 
schlusses jedem Mitgliedstaat möglich, nicht nur dem von der jeweiligen Sanktion betroffenen Mitgliedstaat. Im Rahmen einer solchen Klage kann neben der Sanktion selbst auch immer noch die DurchsetzungsVO als deren rechtliche Grundlage gerügt werden, und zwar auch unter dem Gesichtspunkt des Kompetenzverstoßes. 